\title{
ON THE DENSENESS OF JACOBI POLYNOMIALS
}

\section{SARJOO PRASAD YADAV}

\author{
Received 29 May 2003
}

Let $\mathbf{X}$ represent either a space $\mathbf{C}[-1,1]$ or $\mathbf{L}_{\alpha, \beta}^{p}(w), 1 \leq p<\infty$, of functions on $[-1,1]$. It is well known that $\mathbf{X}$ are Banach spaces under the sup and the $p$-norms, respectively. We prove that there exist the best possible normalized Banach subspaces $\boldsymbol{X}_{\alpha, \beta}^{k}$ of $\boldsymbol{X}$ such that the system of Jacobi polynomials is densely spread on these, or, in other words, each $f \in \mathbf{X}_{\alpha, \beta}^{k}$ can be represented by a linear combination of Jacobi polynomials to any degree of accuracy. Explicit representation for $f \in \mathbf{X}_{\alpha, \beta}^{k}$ has been given.

2000 Mathematics Subject Classification: 41A10, 42C10, 46B25.

1. Introduction. Let $1 \leq p \leq \infty, w(x)=(1-x)^{\alpha}(1+x)^{\beta}, \alpha, \beta>-1, x \in[-1,1]$, and let $\mathbf{L}_{\alpha, \beta}^{p}(w)$ denote the Banach space of functions $f:[-1,1] \rightarrow \mathfrak{R}$ with $\left\|f x^{j} w\right\|_{p}<\infty$, $j=0,1,2, \ldots$. If $p=\infty$, assume $f$ is continuous. Let $\mathbf{X}_{\alpha, \beta}^{k}$ be the linear manifolds of $\mathbf{L}_{\alpha, \beta}^{p}(w)$ with parameters $\alpha, \beta$, and $k$ such that for all $f \in \mathbf{X}_{\alpha, \beta}^{k}$, the following endpoint, called "the pole" condition, is satisfied:

$$
\int_{0}^{t}|f(\cos \varphi)-A| \varphi^{2 \alpha+1} d \varphi=o\left(t^{2 \alpha+2}\right) .
$$

Also, for some $k$, the following additional condition, called "the antipole" condition, is assumed to be satisfied:

$$
\int_{0}^{h}|f(-\cos \varphi)| \varphi^{\beta-\alpha+k} d \varphi=o(1)
$$

It may be noted that the first condition would have been satisfied by $A=f(1)$, where $f$ is continuous at $x=1$. Also, "the antipole" condition (1.2) is weaker than that in [15, Theorem 9.1.4]. Let $\left\{g_{n}\right\}$ be a $c$-sequence in $X_{\alpha, \beta}^{k}$ and let its limit be $g$. Then $g$ will satisfy (1.1) and (1.2), proving that $g \in \mathbf{X}_{\alpha, \beta}^{k}$. Thus, the spaces $\mathbf{X}_{\alpha, \beta}^{k}$ are normalized Banach subspaces of $\mathbf{X} \equiv \mathbf{L}_{\alpha, \beta}^{p}(w)$ with norm

$$
\|f\|_{\mathbf{X}_{\alpha, \beta}^{k}}=c_{1}\|f\|_{\mathbf{X}} \quad\left(c_{1} \geq 1\right) .
$$

In all, we are going to prove that $\mathbf{X}_{\alpha, \beta}^{k}$ for $1 \leq p \leq \infty$ with given $\alpha, \beta$, and $k$ are Banach spaces on which the set of Jacobi polynomials is densely spread for each $p$. In other words, every element of $\mathbf{X}_{\alpha, \beta}^{k}$ is expressible as a linear combination of Jacobi polynomials to any degree of accuracy. Our settings show that the subspaces $\mathbf{X}_{\alpha, \beta}^{k}$ are the best possible in $\mathbf{L}_{\alpha, \beta}^{p}(w)$. Our proof constructed for $j=0$ works for all $j=0,1,2, \ldots$ 
We associate a Fourier-Jacobi expansion for all $f \in \mathbf{X}_{\alpha, \beta}^{k}$ as

$$
f(x) \sim \sum_{n=0}^{\infty} \hat{f}(n) \omega_{n}^{(\alpha, \beta)} R_{n}^{(\alpha, \beta)}(x),
$$

where

$$
\begin{aligned}
\omega_{n}^{(\alpha, \beta)} & =\left(\int_{-1}^{1}\left\{R_{n}^{(\alpha, \beta)}(x)\right\}^{2} w(x) d x\right)^{-1} \\
& =\frac{(2 n+\alpha+\beta+1) \Gamma(n+\alpha+\beta+1) \Gamma(n+\alpha+1)}{2^{\alpha+\beta+1} \Gamma(n+\beta+1) \Gamma(n+1) \Gamma(\alpha+1) \Gamma(\alpha+1)} \\
& \asymp n^{2 \alpha+1} L(n) \quad\left(L(n) \equiv 1+O\left(\frac{1}{n}\right)\right),
\end{aligned}
$$

$\hat{f}(n)$ is the $n$th Fourier-Jacobi transform of $f$ given by

$$
\hat{f}(n)=\int_{-1}^{1} f(x) R_{n}^{(\alpha, \beta)}(x) w(x) d x
$$

$R_{n}^{(\alpha, \beta)}(x)$ is the normalized Jacobi polynomial such that

$$
R_{n}^{(\alpha, \beta)}(x)=\frac{P_{n}^{(\alpha, \beta)}(x)}{P_{n}^{(\alpha, \beta)}(1)},
$$

where $P_{n}^{(\alpha, \beta)}(x)$ is the $n$th Jacobi polynomial of degree $n$ and order $(\alpha, \beta)$ (see [15]).

2. Preliminaries. We choose a linear combination $\sigma_{n}^{k}$ of Jacobi polynomials as

$$
\begin{aligned}
\sigma_{n}^{k}\left(f, \cos \vartheta, X_{\alpha, \beta}^{k}\right)= & c_{n}+c_{n-1} R_{1}^{(\alpha, \beta)}(\cos \vartheta)+c_{n-2} R_{2}^{(\alpha, \beta)}(\cos \vartheta) \\
& +\cdots+c_{0} R_{n}^{(\alpha, \beta)}(\cos \vartheta) \\
\equiv & \sum_{\nu=0}^{n} c_{n-\nu} R_{v}^{(\alpha, \beta)}(\cos \vartheta)
\end{aligned}
$$

where coefficients $c_{i}$ are given by

$$
c_{n-v} \equiv\left(\frac{A_{n-v}^{k}}{A_{n}^{k}}\right) \hat{f}(v) \omega_{v}^{(\alpha, \beta)},
$$

$v=0,1, \ldots, n$ and $n=0,1,2, \ldots A_{i}^{k}(i=0,1,2, \ldots)$ are binomial coefficients of $x^{i}$ in the expansion of $(1-x)^{-k-1}$. Thus, $\sigma_{n}^{k}\left(f, \cos 9, \mathbf{X}_{\alpha, \beta}^{k}\right)$ is the $n$th Cesàro mean of order $k$ of the Fourier-Jacobi expansion given in (1.4) (see [15, page 244]). Recently in [22], we have proved the following result:

$$
\left\|\sigma_{n}^{1}\left(f, \cos \vartheta, \mathbf{X}_{\alpha, \beta}^{1}\right)-f(\cos \vartheta)\right\|_{\mathbf{X}_{\alpha, \beta}^{1}} \longrightarrow 0 \quad \text { as } n \longrightarrow \infty,
$$

for the case $k=1$ under only the saturation-type condition (1.1) at the pole $x=1$ for $\alpha \geq \beta=-1 / 2$ and $\alpha \geq \beta>-1 / 2$ with $\alpha+\beta \leq 0$. The case $\alpha \geq \beta>-1 / 2$ with $\alpha+\beta>0$ 
has also been solved in [18]. The best possible cases of general order $k$ have not been handled so far. We settle the problem by proving

$$
\left\|\sigma_{n}^{k}\left(f, \cos \vartheta, \mathbf{X}_{\alpha, \beta}^{k}\right)-f(\cos \vartheta)\right\|_{\mathbf{X}_{\alpha, \beta}^{k}} \longrightarrow 0
$$

as $n \rightarrow \infty$, by deciding the best possible span of $k$ for $\alpha \geq \beta \geq-1 / 2$. We settle the complete problem in four steps given as Theorems 3.1, 3.2, 3.3, and 3.4. The subject approximation of functions in terms of polynomials which indicate its denseness is an important part of analysis with many notable contributions, such as Riesz [14], Pollard [11, 12, 13], Newman and Rudin [10], Askey [1, 2], Badkov [3], Nevai [9], Máté et al. [7], $\mathrm{Xu}[16,17]$, Lasser and Obermaier [5], Li [6], Mhaskar [8], Yadav [18, 19, 20, 21, 22]. The central ideas used in our proofs are the convolution structure for Jacobi series [2], and endpoint convergence of Fourier-Jacobi expansions [15].

3. Main results. We prove the following with prior assumption that $\alpha \geq \beta \geq-1 / 2$.

THEOREM 3.1. Statement (2.4) holds true for $k \geq \alpha+\beta+1$ only if the pole condition (1.1) is satisfied.

THEOREM 3.2. For $\beta>-1 / 2, \alpha+1 / 2<k<\alpha+\beta+1$, statement (2.4) is true if "the pole" condition (1.1) and "the antipole" condition (1.2) are satisfied.

THEOREM 3.3. For $k<\alpha+1 / 2$, statement (2.4) is not true, that is, there exist functions $f \in \mathbf{X}_{\alpha, \beta}^{k}$ so that the norm in (2.4) tends to infinity.

THEOREM 3.4. For $\alpha+1 / 2<k<\alpha+\beta+1$, but without "the antipole" condition (1.2), statement (2.4) is not true. That is, there exist functions not satisfying (1.2), having their kth Cesàro transform divergent.

4. Results to be used. The following formulae and lemmas have been used to complete the proofs of our theorems:

$$
P_{n}^{(\alpha, \beta)}(1)=\left(\begin{array}{c}
n+\alpha \\
n
\end{array}\right)
$$

(see $[15,(4.1 .1)]) ;$

$$
P_{n}^{(\alpha, \beta)}(-x)=(-1)^{n} P_{n}^{(\beta, \alpha)}(x)
$$

(see [15, (4.1.3)]). For $\alpha, \beta$ arbitrary reals, $c$ a fixed positive number, and $n \rightarrow \infty$,

$$
P_{n}^{(\alpha, \beta)}(\cos \theta)= \begin{cases}\theta^{-\alpha-1 / 2} O\left(n^{-1 / 2}\right), & \frac{c}{n} \leq \theta \leq \frac{\pi}{2}, \\ O\left(n^{\alpha}\right), & 0 \leq \theta \leq \frac{c}{n}\end{cases}
$$

(cf. [15, (7.32.5)]), and

$$
P_{n}^{(\alpha, \beta)}(\cos \theta)=O\left(n^{-1 / 2}\right)
$$

for $0<\theta \leq \pi / 2, \alpha \leq-1 / 2$ (cf. [15, (7.32.7)]). 
For $\alpha>-1, \beta>-1$, and $c / n \leq \theta \leq \pi-c / n$,

$$
P_{n}^{(\alpha, \beta)}(\cos \theta)=n^{-1 / 2} K(\theta)\left\{\cos (N \theta+\gamma)+O(n \sin \theta)^{-1}\right\}
$$

(see [15, Theorem 8.21.13]) and

$$
P_{n}^{(\alpha, \beta)}(\cos \theta)=n^{-1 / 2} K(\theta) \cos (N \theta+\gamma)+O\left(n^{-3 / 2}\right)
$$

for $\alpha, \beta$ arbitrary and $0<\theta<\pi$ (see [15, Theorem 8.21.8]), where

$$
K(\theta)=\pi^{-1 / 2}\left(\frac{\sin \theta}{2}\right)^{-\alpha-1 / 2}\left(\frac{\cos \theta}{2}\right)^{-\beta-1 / 2}, \quad N=n+\frac{\alpha+\beta+1}{2},
$$

and $\gamma=-(\alpha+1 / 2) \pi / 2$. The error term in (4.6) holds uniformly in the interval $[\epsilon, \pi-\epsilon]$, for $\epsilon>0$. These formulae have been indirectly used with division by

$$
\left[P_{n}^{(\alpha, \beta)}(1)=\left(\begin{array}{c}
n+\alpha \\
n
\end{array}\right)\right]
$$

to substitute the value of $R_{n}^{(\alpha, \beta)}(\cos \varphi)$ in the following lemmas which are crucial in the proofs of Theorems 3.1, 3.2, 3.3, and 3.4.

LEMMA 4.1. Let $f \in \mathbf{X}_{\alpha, \beta}^{k}$. Then, as $n \rightarrow \infty$,

$$
\int_{0}^{\pi} F(\varphi) R_{n}^{(\alpha+k+1, \beta)}(\cos \varphi) d \varphi= \begin{cases}o\left(n^{-2 \alpha-2}\right), & \text { for } k>\alpha+\frac{1}{2} \\ o\left(n^{-\alpha-k-3 / 2} \log n\right), & \text { for } k=\alpha+\frac{1}{2} \\ o\left(n^{-\alpha-k-3 / 2}\right), & \text { for } k<\alpha+\frac{1}{2}\end{cases}
$$

where

$$
F(\varphi)=\{f(\cos \varphi)-A\}\left(\frac{\sin \varphi}{2}\right)^{2 \alpha+1}\left(\frac{\cos \varphi}{2}\right)^{2 \beta+1},
$$

provided that, for $\beta>-1 / 2$ and $\alpha+1 / 2<k<\alpha+\beta+1$, the antipole condition (1.2) is satisfied. For $-1<\beta \leq-1 / 2$ (or $\beta>-1 / 2$ but $k \geq \alpha+\beta+1$ ), no antipole condition is necessary.

Proof of Lemma 4.1. Let $n$ be large enough and

$$
\int_{0}^{\pi} F(\varphi) R_{n}^{(\alpha+k+1, \beta)}(\cos \varphi) d \varphi=\int_{0}^{c / n}+\int_{c / n}^{\delta}+\int_{\delta}^{\pi-\delta}+\int_{\pi-\delta}^{\pi-c / n}+\int_{\pi-c / n}^{\pi}=\sum_{i=1}^{5} T_{i}
$$

where $c, \delta$, and $\delta$ are small but fixed positive reals. Now, the proof of the lemma follows upon estimating (4.11) on the lines of [22, Lemma 1] using orders of $R_{n}^{(\alpha+k+1, \beta)}(\cos \varphi)$, the pole condition (1.1), and the antipole condition (1.2). Thus, the proof of Lemma 4.1 is complete. 
We denote the $n$th Cesàro mean of order $k$ of the series (1.4) by $\sigma_{n}^{k}\left(f, \cos \vartheta, X_{\alpha, \beta}^{k}\right)$ defined by (2.1). Lemma 4.2, which is basic in the proofs of Theorems 3.1, 3.2, 3.3, and 3.4 , provides new convergence criteria for Jacobi series at the endpoints of the interval $[-1,1]$ (see [21]).

LEMMA 4.2. If $f \in \mathbf{X}_{\alpha, \beta}^{k}(\alpha>-1, \beta>-1)$, then the series (1.4) is $(C, k)$-summable to A at $x=1$, for $k>\alpha+1 / 2$, or

$$
\lim _{n \rightarrow \infty} \sigma_{n}^{k}\left(f, \cos \vartheta, X_{\alpha, \beta}^{k}\right)-A=0
$$

at $\vartheta=0$, for $k>\alpha+1 / 2$, provided that in the case

$$
\beta>-\frac{1}{2}, \quad \alpha+\frac{1}{2}<k<\alpha+\beta+1,
$$

the antipole condition (1.2) is satisfied. (For $-1<\beta \leq-1 / 2$ or for $k \geq \alpha+\beta+1$, no antipole condition is necessary.) For $k \leq \alpha+1 / 2$ or for $k>\alpha+1 / 2$, but without the antipole condition (1.2), statement (4.12) is not true.

Proof of Lemma 4.2. We have

$$
\sigma_{n}^{k}(x)=\left(A_{n}^{k}\right)^{-1} \sum_{\nu=0}^{n} A_{n-v}^{k} \hat{f}(\nu) \omega_{\nu}^{(\alpha, \beta)} R_{\nu}^{(\alpha, \beta)}(x) .
$$

If $A$ is any constant, then

$$
\sigma_{n}^{k}(x)-A=\left(A_{n}^{k}\right)^{-1} \sum_{\nu=0}^{n} A_{n-v}^{k-1}\left[\sum_{i=0}^{v} \hat{f}(\nu) \omega_{i}^{(\alpha, \beta)} R_{i}^{(\alpha, \beta)}(x)-A\right] .
$$

Using (1.5), we get, at $x=1$,

$$
\begin{aligned}
\sigma_{n}^{k}(1)-A= & \left(A_{n}^{k}\right)^{-1} \sum_{\nu=0}^{n} A_{n-v}^{k-1}\left[\sum_{i=0}^{v} \hat{f}(\nu) \omega_{i}^{(\alpha, \beta)} R_{i}^{(\alpha, \beta)}(1)-A\right] \\
= & \int_{0}^{\pi}\{f(\cos \varphi)-A\}\left(A_{n}^{k}\right)^{-1} \sum_{\nu=0}^{n} \frac{\alpha+1}{2 \nu+\alpha+\beta+1} \\
& \cdot A_{n-\nu}^{k-1} \omega_{\nu}^{(\alpha+1, \beta)} R_{v}^{(\alpha+1, \beta)}(\cos \varphi) \rho^{(\alpha, \beta)}(\varphi) d \varphi,
\end{aligned}
$$

where

$$
\rho^{(\alpha, \beta)}(\varphi) \equiv\left(\frac{\sin \varphi}{2}\right)^{2 \alpha+1}\left(\frac{\cos \varphi}{2}\right)^{2 \beta+1}
$$

by the use of the summation formula of Szegö [15, page 71] and the orthogonality of Jacobi polynomials. But the right-hand side of (4.14) tends to zero under the conditions of Lemma 4.2 as detailed in [21, 22]. Thus, the proof of Lemma 4.2 is complete. 


\section{Proofs}

Proof of Theorem 3.1. We substitute $\Delta \lambda_{n, v}=A_{n-v}^{k-1} / A_{n}^{k}$ for $v \leq n$ and zero otherwise in [19, equation (2.11)] to get

$$
\begin{aligned}
& \sigma_{n}^{k}\left(f,(\cos \vartheta), \mathbf{X}_{\alpha, \beta}^{k}\right)-f(\cos \vartheta) \\
& =\int_{0}^{\pi}\left[T_{\psi} f(\cos \vartheta)-f(\cos \vartheta)\right] \sum_{\nu=0}^{n} \frac{\alpha+1}{2 v+\alpha+\beta+2} \frac{A_{n-v}^{k-1}}{A_{n}^{k}} \omega_{\nu}^{(\alpha+1, \beta)} R_{\nu}^{(\alpha+1, \beta)}(\cos \psi) \rho^{(\alpha, \beta)}(\psi) d \psi,
\end{aligned}
$$

where $T_{\psi} f(\cos 9)$ is the generalized translate of $f(\cos 9)$ in the interval $[0, \pi]$ (see [2]). Also,

$$
\left\|T_{\psi} f\right\|_{X} \leq\|f\|_{X}
$$

for $\alpha \geq \beta \geq-1 / 2$, and

$$
\lim _{\psi \rightarrow 0} T_{\psi} f=f
$$

in the sense of strong limit. (See Bavinck [4, page 770].) But

$$
\left\|T_{\psi} f-f\right\|_{X} \leq\left\|T_{\psi} f-A\right\|_{X}+\|f-A\|_{X} \leq A_{6}\|f-A\|_{X}
$$

for some nonnegative constant $A_{6}$. The constant $A_{6}$ will be independent of $f$ by the Banach-Steinhaus theorem. Also,

$$
\left\|T_{\psi} f-f\right\|_{X} \geq\left\|T_{\psi} f-A\right\|_{X}-\|f-A\|_{X} \geq A_{7}\|f-A\|_{X} .
$$

Again, $A_{7}$ will be independent of $f$. Thus, we have the asymptotic equality

$$
\left\|T_{\psi} f-f\right\|_{X} \simeq\|f-A\|_{X} .
$$

Thus, we compare (5.1) and (4.14) to get

$$
\left\|\sigma_{n}^{k}\left(f, \cos \vartheta, \mathbf{X}_{\alpha, \beta}^{k}\right)-f(\cos \vartheta)\right\|_{\mathbf{X}_{\alpha, \beta}^{k}} \leq A_{8}\left\|\sigma_{n}^{k}\left(f, 1, \mathbf{X}_{\alpha, \beta}^{k}\right)-A\right\|_{\mathbf{X}_{\alpha, \beta}^{k}}
$$

for $\alpha \geq \beta \geq-1 / 2$ and an absolute constant $A_{8}$. But the right-hand side of this inequality tends to zero as $n$ tends to $\infty$ by Lemma 4.2 , for $k \geq \alpha+\beta+1$. Thus, statement (2.4) follows. This completes the proof of Theorem 3.1.

Proof of TheOrem 3.2. For $\beta>-1 / 2, \alpha+1 / 2<k<\alpha+\beta+1$, statement (4.12) holds if the antipole condition (1.2) is satisfied, that is, in Theorem 3.2, for all $f \in \mathbf{X}_{\alpha, \beta}^{k}$ satisfying both linear conditions (1.1) and (1.2). Thus, the arguments of the proof of Theorem 3.1 apply to the proof of Theorem 3.2 and statement (2.4) holds in this case also. This completes the proof of Theorem 3.2.

Proof of Theorem 3.3. It is well known that (see Szegö [15, equation (9.41.17), page 262]) there exist continuous functions such that for $k=\alpha+1 / 2$,

$$
\sigma_{n}^{k}\left(f, 1, \mathbf{X}_{\alpha, \beta}^{k}\right)>C \log n \quad(C>0) .
$$


This is sufficient to conclude, because of the regularity that there exist functions $f \in$ $\mathbf{X}_{\alpha, \beta}^{k}$ such that

$$
\left\|\sigma_{n}^{k}\left(f, \cos \vartheta, \mathbf{X}_{\alpha, \beta}^{k}\right)-f(\cos \vartheta)\right\|_{\mathbf{X}_{\alpha, \beta}^{k}} \longrightarrow \infty
$$

for $k \leq \alpha+1 / 2$. Thus, in this case, statement (2.4) is not true. This completes the proof of Theorem 3.3.

Proof of Theorem 3.4. Here, by an example, we show that there exist functions such that the sequence $\left\{\sigma_{n}^{k}(1)\right\}$ given in (4.14) diverges showing that statement (2.4) is not true. We consider a function $f(x)=(1+x)^{\mu}$ given in [15, page 265]. Its Jacobi series at the endpoint $x=1$ is

$$
\begin{aligned}
& \sum_{n=0}^{\infty} \omega_{n}^{(\alpha, \beta)} \int_{-1}^{1}(1-x)^{\alpha}(1+x)^{\beta+\mu} R_{n}^{(\alpha, \beta)}(x) R_{n}^{(\alpha, \beta)}(1) d x \\
& \equiv \sum_{n=0}^{\infty}(-1)^{n} \omega_{n}^{(\alpha, \beta)} \int_{-1}^{1}(1-x)^{\alpha+\mu}(1+x)^{\beta} R_{n}^{(\beta, \alpha)}(x) d x
\end{aligned}
$$

The principal part of the general term of the series (5.10) is approximately

$$
(-1)^{n} n^{\alpha-\beta-2 \mu-1} \quad \text { or } \quad(-1)^{n} A_{n}^{\alpha-\beta-2 \mu-1}
$$

as $R_{n}^{(\alpha, \beta)}(1)=1$. The expansion in (5.10) holds good for $\mu+\beta>-1$ or $-\beta-1<\mu$ (see [15, page 265]), but the antipole condition (1.2) is not satisfied if $\mu \leq 1 / 2(\alpha-\beta-k-1)$. So, for $-\beta-1<\mu \leq 1 / 2(\alpha-\beta-k-1)$, the Fourier-Jacobi series exists for the function $f(x)=$ $(1+x)^{\mu}$, but it does not satisfy the antipole condition (1.2). Also, for $k \leq(\alpha-\beta-2 \mu-1)$, the expansion in (5.10) is not $(C, k)$-summable for $\beta>-1 / 2, \alpha+1 / 2<k<\alpha+\beta+1$ (see [15, page 265]). Thus, the sequence given in (4.14) diverges, which, by (5.1), leads to the conclusion that statement (2.4) is not true. This proves Theorem 3.4.

ACKNOWLEDGMENT. The author expresses his gratitude to both referees of the paper for their kind perusal.

\section{REFERENCES}

[1] R. Askey, Mean convergence of orthogonal series and Lagrange interpolation, Acta Math. Acad. Sci. Hungar. 23 (1972), 71-85.

[2] R. Askey and S. Wainger, A convolution structure for Jacobi series, Amer. J. Math. 91 (1969), 463-485.

[3] V. M. Badkov, Convergence in the mean and almost everywhere of Fourier series in polynomials that are orthogonal on an interval, Mat. Sb. (N.S.) 95(137) (1974), 229-262.

[4] H. Bavinck, A special class of Jacobi series and some applications, J. Math. Anal. Appl. 37 (1972), 767-797.

[5] R. Lasser and J. Obermaier, On the convergence of weighted Fourier expansions with respect to orthogonal polynomials, Acta Sci. Math. (Szeged) 61 (1995), no. 1-4, 345-355.

[6] Zh.-K. Li, Approximation of the Jacobi-Weyl class of functions by the partial sums of Jacobi expansions, Approximation Theory and Function Series (Budapest, 1995), Bolyai Soc. Math. Stud., vol. 5, János Bolyai Math. Soc., Budapest, 1996, pp. 247-258. 
[7] A. Máté, P. Nevai, and V. Totik, Necessary conditions for weighted mean convergence of Fourier series in orthogonal polynomials, J. Approx. Theory 46 (1986), no. 3, 314322.

[8] H. N. Mhaskar, Introduction to the Theory of Weighted Polynomial Approximation, Series in Approximations and Decompositions, vol. 7, World Scientific Publishing, New Jersey, 1996.

[9] P. G. Nevai, Orthogonal polynomials, Mem. Amer. Math. Soc. 18 (1979), no. 213, v+185.

[10] J. Newman and W. Rudin, Mean convergence of orthogonal series, Proc. Amer. Math. Soc. 3 (1952), 219-222.

[11] H. Pollard, The mean convergence of orthogonal series. I, Trans. Amer. Math. Soc. 62 (1947), 387-403.

[12] _ The mean convergence of orthogonal series. II, Trans. Amer. Math. Soc. 63 (1948), 355-367.

[13] _ The mean convergence of orthogonal series. III, Duke Math. J. 16 (1949), 189-191.

[14] M. Riesz, Sur les functions conjugées, Math. Z. 27 (1927), 218-244 (French).

[15] G. Szegö, Orthogonal Polynomials, 3rd ed., vol. 23, American Mathematical Society, Rhode Island, 1967.

[16] Y. Xu, Mean convergence of generalized Jacobi series and interpolating polynomials. I, J. Approx. Theory 72 (1993), no. 3, 237-251.

[17] _ Mean convergence of generalized Jacobi series and interpolating polynomials. II, J. Approx. Theory 76 (1994), no. 1, 77-92.

[18] S. P. Yadav, Some Lebesgue subspaces approximable by Jacobi polynomials, to appear.

[19] _ On the saturation order of approximation processes involving Jacobi polynomials, J. Approx. Theory 58 (1989), no. 1, 36-49.

[20]_ Saturation orders of some approximation processes in certain Banach spaces, Studia Sci. Math. Hungar. 28 (1993), no. 3-4, 1-18.

[21] _ On the generalized summability theorem of G. Szegö for Jacobi series, Ganita Sandesh 12 (1998), no. 1-2, 55-64.

[22] _ On a Banach space approximable by Jacobi polynomials, Acta Math. Hungar. 98 (2003), no. 1-2, 21-30.

Sarjoo Prasad Yadav: Department of Mathematics/ Computer Applications, Government Model Science College, APS University, 486001 Rewa, Madhya, India

E-mail address: yadavsp@sancharnet.in 


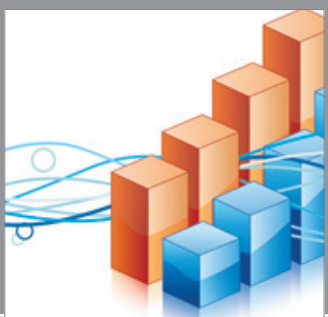

Advances in

Operations Research

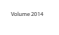

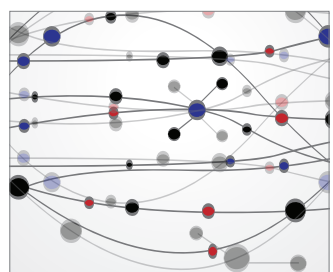

\section{The Scientific} World Journal
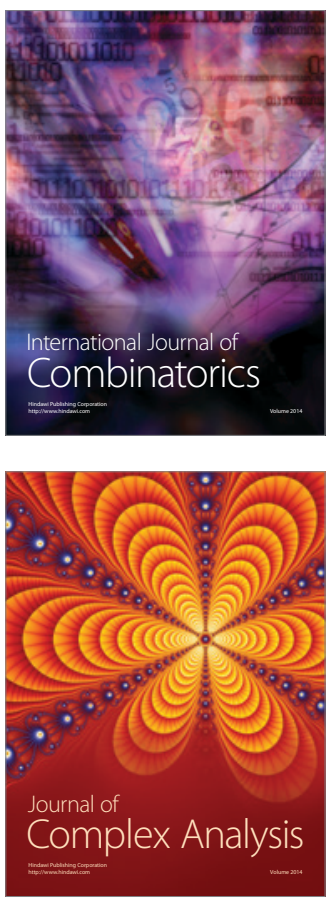

International Journal of

Mathematics and

Mathematical

Sciences
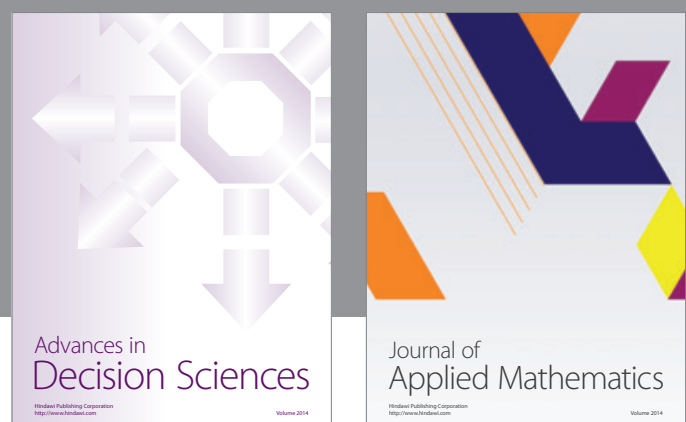

Journal of

Applied Mathematics
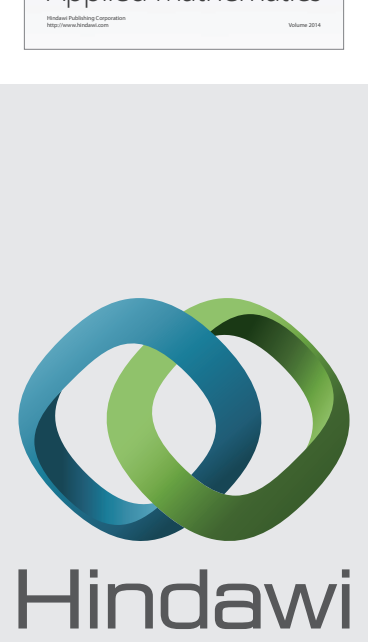

Submit your manuscripts at http://www.hindawi.com
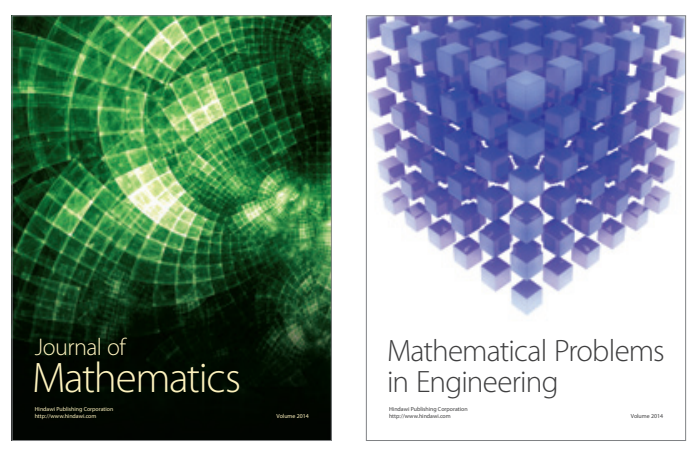

Mathematical Problems in Engineering
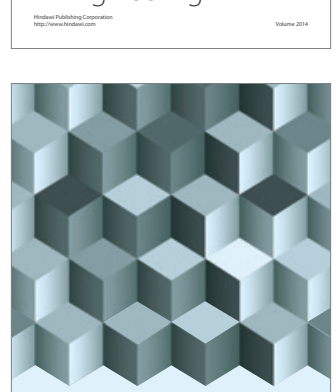

Journal of

Function Spaces
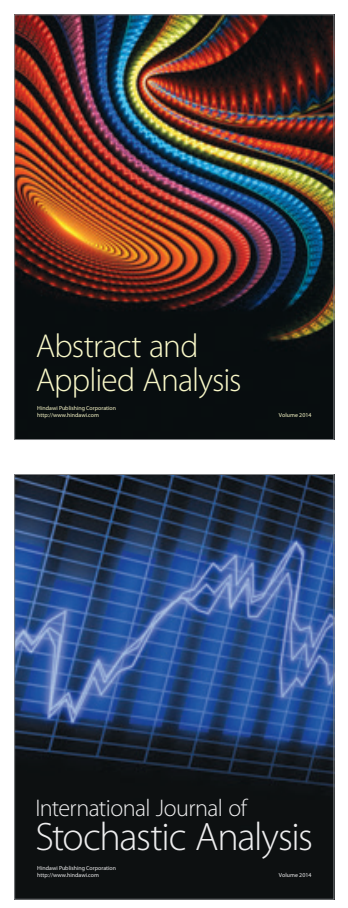

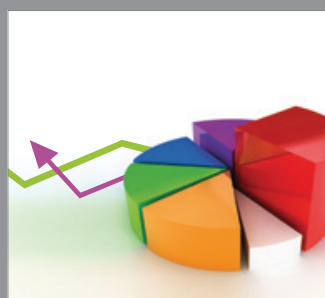

ournal of

Probability and Statistics

Promensencen
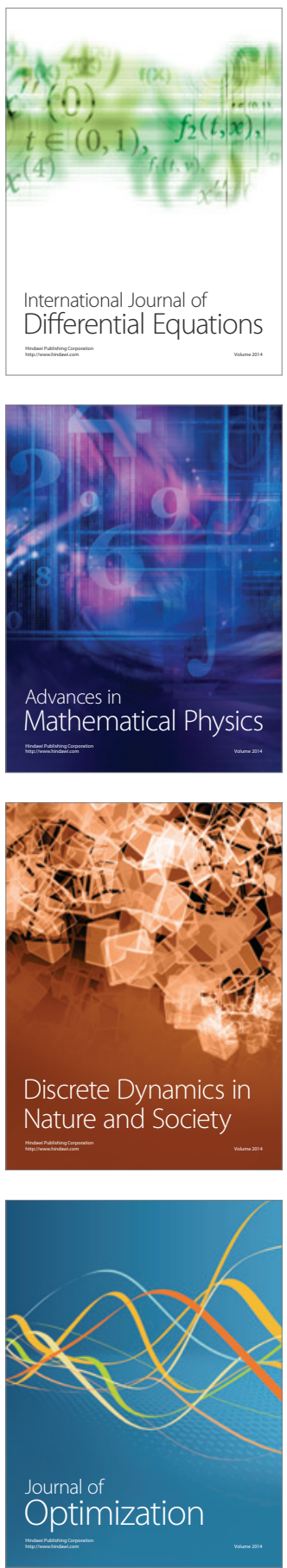